\title{
POLYNOMIAL ITERATIONS TO ROOTS \\ OF ALGEBRAIC EQUATIONS
}

\author{
ALSTON S. HOUSEHOLDER
}

If $\xi$ is a root of the equation

$$
f(x)=0,
$$

and if $\phi(x)$ satisfies

$$
\phi(\xi)=\xi, \quad \phi^{(s)}(\xi)=0 \quad(s=1,2, \cdots, r-1),
$$

then $\phi(x)$ is said to define an iteration of order $r$ to the root $\xi$. In fact, for $r>1$, when $x_{0}$ is in a sufficiently small neighborhood of $\xi$ the sequence

$$
x_{i+1}=\phi\left(x_{i}\right)
$$

converges to $\xi$ with

$$
x_{i+1}=\xi+O\left(x_{i}-\xi\right)^{r} .
$$

For analytic $f$, iterations of all orders exist and can be constructed in many ways. Domb $[2]^{1}$ has shown further that for polynomial $f$ it is always possible to make $\phi$ a polynomial. The purpose of this note is to describe a simple algorithm:

Let $f(x)$ be a polynomial with no multiple factors; let $p(x)$ and $q(x)$ be any polynomials satisfying

$$
p f^{\prime}+q f \equiv 1
$$

let

$$
\begin{array}{ll}
\phi_{1}=x-p_{1} f, & \phi_{r}=\phi_{r-1}+p_{r}(-f)^{r} / r !, \\
p_{1}=p, & p_{r}=p p_{r-1}^{\prime}-(r-1) q p_{r-1} .
\end{array}
$$

Then $\phi_{r}(x)$ is clearly a polynomial, and it defines an iteration of order $r+1$ to any root $\xi$ of the equation (1).

While a direct induction is possible, it is simpler to proceed otherwise. Schröder [3] (see also [1]) has shown that

$$
\begin{aligned}
\phi_{r} & =x+\sum_{1}^{r}(-F)^{\nu} \delta^{\nu-1} F / \nu !, \\
\delta^{0} F & =1 / F^{\prime}, \quad \delta^{\nu+1} F=\left(\delta^{\nu} F\right)^{\prime} / F^{\prime},
\end{aligned}
$$

Presented to the Society, November 25, 1950; received by the editors October 23, 1950.

${ }^{1}$ Numbers in brackets refer to the bibliography at the end of the paper. 
defines an iteration of order $r+1$ to the roots of

$$
F=0 \text {. }
$$

We ask, then, whether a function $g(x)$ can be found such that for

$$
F=g f
$$

the functions $\phi_{r}$ defined by (7) are polynomials. For $r=1$ we require that

$$
F / F^{\prime}=p f
$$

for some polynomial $p$. To achieve this it is sufficient to choose $p$ and $f$ to satisfy (5) while

$$
g^{\prime} / g=q / p .
$$

Suppose, now, it has been verified that

$$
F^{r} \delta^{r-1} F=p_{r} f^{r},
$$

or, by (8), that

$$
\delta^{r-1} F=p_{r} g^{-r}
$$

Then

$$
\delta^{r} F=\left(p_{r}^{\prime} g^{-r}-r p_{r} g^{\prime} g^{-r-1}\right) / F^{\prime},
$$

and after a few manipulations

$$
F^{r+1} \delta^{r} F=f^{r+1}\left(p p_{r}^{\prime}-r q p_{r}\right) .
$$

Hence

$$
p_{r+1}=p p_{r}^{\prime}-r q p_{r}
$$

and the induction is complete.

\section{BIBLIOGRAPHY}

1. D. R. Blaskett and H. Schwerdtfeger, $A$ formula for the solution of an arbitrary analytic equation, Quarterly of Applied Mathematics vol. 3 (1945) pp. 266-268.

2. C. Domb, On iterative solutions of algebraic equations, Proc. Cambridge Philos. Soc. vol. 45 (1949) pp. 237-240.

3. Ernst Schröder, Über unendliche viele Algorithmen zur Auflösung der Gleichungen, Math. Ann. vol. 2 (1870) pp. 317-365.

OAK Ridge National Laboratory 